\title{
La littérature française comme discipline académique dans la Roumanie de l'entre-deux-guerres. Le cas de Charles Drouhet
}

\author{
Dragos Jipa
}

Faculté des Langues et Littératures Étrangères, Université de Bucarest*

Cet article étudie les recherches sur la littérature française et son enseignement dans la Roumanie de l'entre-deux-guerres, avec un double regard : en suivant les concepts et les discours et en restituant leurs cadres institutionnels et (inter)nationaux. À partir du cas de Charles Drouhet, professeur à l'Université de Bucarest, l'article essaie de montrer comment l'histoire littéraire et la littérature comparée exportées de France en Roumanie ont fourni les critères d'évaluation pour les recherches et les méthodes pour l'enseignement de la littérature française au niveau universitaire. De plus, la dimension nationale qui était attribuée à l'enseignement de la littérature française au niveau secondaire permet de mettre au jour la position de Drouhet, promoteur d'une autonomie du savoir face aux interférences du politique. Vue d'aujourd'hui, cette histoire permet de comprendre plusieurs étapes d'évolution de la discipline en Roumanie jusqu'à la fin du $\mathrm{xx}^{\mathrm{e}}$ siècle.

Mots-clés : littérature française, enseignement, recherche, institution, histoire littéraire, littérature comparée, nation.

Ce texte propose une étude historique de la littérature française en tant que discipline académique dans la Roumanie de l'entre-deux-guerres. À partir d'un cas individuel, celui du professeur Charles Drouhet, qui a enseigné pendant plus de deux décennies à l'université de la capitale, l'analyse propose quelques observations à partir de cet objet d'étude, tel qu'on peut le (re)construire en resituant l'activité de l'individu dans son époque ${ }^{1}$. Elle a ainsi pour objectif non seulement

\footnotetext{
*dragos.jipa@g.unibuc.ro

1 Pour une discussion sociologique de la discipline comme « un corps de connaissances inscrit dans des textes, des exemples paradigmatiques et des formes d'instrumentation, qui fait l'objet d'une transmission pédagogique, ce qui nécessite une mise en forme, ou confirmation du savoir à des fins d'inculcation, une gradation des traductions pédagogiques du corpus et un programme d'enseignement », voir Fabiani 2006. Pour quelques réflexions méthodologiques en vue d'une
} 
de montrer en quoi le cas de Drouhet est représentatif pour la discipline dans les années 1920-1930, mais aussi d'offrir quelques éléments qui permettent de comprendre la situation contemporaine des études sur la littérature française en Roumanie, qui s'inscrivent dans une histoire souvent oubliée.

Comme toute étape d'une évolution historique plus longue, l'activité de Drouhet a subi les deux « lectures » habituelles : d'une part, avec la transformation ultérieure des discours sur la littérature, notamment sous l'influence du mouvement structuraliste, son activité a été critiquée et considérée comme désuète ; d'autre part, ses innovations en matière d'enseignement de la littérature se sont répandues jusqu'à la "naturalisation », au point que, depuis son époque, on a continué dans sa lignée sans en avoir toujours eu conscience. En allant au-delà de ces représentations, cette étude se propose d'abord de contribuer à une histoire de la discipline, en prêtant une attention particulière aux sujets de recherche et aux méthodes d'enseignement. De plus, dans un présent en évolution (qu'on pense à la fameuse " crise » de la littérature, accompagnée d'une crise des études littéraires : voir Schaeffer 2011), revisiter la discipline, se situer à l'époque où les méthodes et les principes aujourd'hui contestés représentaient des nouveautés et étaient en cours de diffusion, permettrait également de comprendre le poids que l'histoire joue encore dans nos pratiques et dans nos attitudes. Une autre précision est encore nécessaire : si l'entre-deux-guerres représente dans l'histoire européenne (et nationale) une époque de domination de la culture française, certes avec des contestations, mais qui ne pouvaient pas encore subvertir l'autorité qu'elle s'était construite depuis des siècles, notre époque voit le « rayonnement de la culture française » plutôt comme un objet d'histoire. Ainsi, les recherches sur la littérature française, tout comme son enseignement, se situent à présent, en Roumanie et dans la région, dans une autre configuration historique et dans un autre « rapport de forces ", différent de celui qui avait contribué à la formation et à la mise en place de cette tradition disciplinaire. Restituer l'historicité des approches, des méthodes, des croyances qui sont arrivées à constituer le fondement de la discipline, ce que Thomas Kuhn appelle la "science normale », est aussi une manière de comprendre les études françaises comme des études historiquement situées, mais qui, par leur prétention à la généralité, essaient toujours d'effacer, de faire disparaître les circonstances de leur pratique effective.

Dans la Roumanie de l'entre-deux-guerres, cette pratique a été représentée d'une manière très importante par Charles Drouhet, le titulaire de la chaire de Langue et littérature françaises à l'Université de Bucarest. Né en Roumanie, en 1879, d'un père français et d'une mère roumaine, Drouhet fait son doctorat à Paris et occupe, entre 1915 et 1940 (année de sa mort), la chaire mentionnée dans l'université la plus importante du pays, fondée au milieu du XIX siècle.

recherche sur la littérature française en tant que discipline académique en Roumanie, voir Jipa 2018. 
L'organisation des facultés selon le principe des chaires avec titulaire (ce n'est que la réforme de l'enseignement de 1948 qui introduira l'organisation en départements) a permis à Drouhet d'asseoir dans son champ disciplinaire une autorité et une légitimité académique qu'il a su renforcer par une activité prodigieuse, que ce soit dans la recherche proprement dite, dans l'enseignement à l'Université ou encore au niveau secondaire, par les manuels de lycée dont il est l'auteur. Ces trois volets de son activité représentent autant de points d'entrée dans cette réflexion sur l'histoire de la discipline. Au sujet de ses recherches, celles qui ont eu comme résultat non seulement sa thèse de doctorat, mais aussi ses publications ultérieures (ouvrages, articles), la question qui s'impose vise les critères de cette activité, et notamment les objets, les concepts et les méthodes qu'il choisit pour que sa recherche soit reconnue comme légitime, dans les multiples champs par rapport auxquels il se situe. Quant à son enseignement universitaire, nous portons un intérêt particulier à la manière dont il construit la littérature française en tant qu'objet de transmission/traduction didactique à l'intention des étudiants de la Faculté de Philosophie et de Lettres, ce qui suppose une série de choix, de sélections, d'omissions, dans ce qu'on pourrait appeler le corpus de la littérature, ainsi que dans les méthodes qui étaient disponibles pour cette opération. Pour ce qui est de l'enseignement secondaire, niveau dans lequel il n'a jamais effectivement enseigné (mais pour lequel il formait la majorité de ses étudiants), ses manuels soulèvent des questions similaires. Toutefois, il faudrait à ce niveau penser au public plus large auquel il s'adressait, tous les élèves roumains, vu que le français était la seule langue étrangère obligatoire dans l'enseignement secondaire de l'époque.

\section{Qu'est-ce qu'une recherche légitime, entre la France et la Roumanie?}

Dans l'article consacré à l'activité du maître, dans le volume de Mélanges d'histoire littéraire et de littérature comparée publié en 1940, Basil Munteanu, disciple et successeur de Charles Drouhet, partage les recherches de celui-ci en deux étapes nettement limitées dans le temps : une première, «strictement française », et une seconde, franco-roumaine (voir Munteanu 1940). Cette délimitation, faite assez naturellement selon le critère thématique de ses intérêts, conduit pourtant à un double effacement : d'une part, même si les sujets de recherche diffèrent, il s'agit d'un même individu qui accumule une formation et un savoir-faire qui, à leur tour, vont progressivement orienter ses activités ; d'autre part, la séparation fait l'impasse sur le contexte intellectuel de ses recherches, un contexte fait de deux champs académiques, français et roumain, entre lesquels il fait non seulement figure de médiateur, mais qu'il met à profit alternativement pour ac- 
croître son capital scientifique (sur les rapports historiques entre la Roumanie et la France, voir entre autres Țurcanu 2006).

En France, son ouvrage le plus important est une « étude critique d'histoire littéraire » sur François Maynard, poète de la première partie du XVII ${ }^{\mathrm{e}}$ siècle, au sujet duquel il donne une étude qui se veut définitive (Drouhet 1909). Il s'agit d'une monographie faite selon le modèle sainte-beuvien de "l'homme et l'œuvre ", mais avec toutes les exigences de méthode de l'histoire littéraire qui avaient été définies par Gustave Lanson et ses disciples (voir Compagnon 1983). D'ailleurs, l'ouvrage - résultat d'un séjour de cinq ans en France - a été présenté comme thèse principale à l'Université de Paris Sorbonne en 1909 et couronné du Prix Bordin par l'Académie française.

Ce cadre institutionnel explique en grande mesure les caractéristiques de son étude, commentée après publication dans plusieurs revues françaises, dont la fameuse Revue d'histoire littéraire de la France, qui reprend dans un compterendu le rapport de la soutenance établi par Gustave Lanson lui-même (pour les citations suivantes, voir Lanson 1910). Selon le professeur de la Sorbonne, l'ouvrage de Drouhet a tous les traits qui avaient été élevés au rang de critères de validité par les partisans des « humanités modernes » (voir Compagnon 1983) : l'usage des sources inédites ( «la biographie de son personnage est jalonnée de toute une série de pièces d'archives, dates et faits, qu'il a eu le mérite de découvrir ») et leur traitement adéquat ( «il fait la critique de ses documents, en général, avec sureté et précision »), la description de l'œuvre et sa situation par rapport aux autres écrivains de son époque (« Sur l'œuvre de Maynard, sur ses caractères et son rapport au mouvement littéraire, il a su et il a dit presque tout ce qu'il faut savoir »). Lorsqu'il s'agit de faire des critiques, Lanson identifie des tendances qui ne concordent pas avec son point de vue, qualifié depuis comme " positiviste » : « Il est toujours délicat d'évaluer et d'interpréter la valeur biographique des poésies amoureuses, de faire le départ du réel et de la fiction, du vécu et de l'imaginé, du traditionnel et du personnel. L'histoire que Drouhet nous conte des amours de Maynard est en très grande partie un roman arbitrairement construit par lui ».

Les autres travaux de l'auteur consistent à cette époque en une chronologie de la correspondance de François Maynard, une étude d'attribution consacrée au même auteur ainsi qu'une étude d'identification des personnages réels qui ont fait l'objet d'un pamphlet de J.L. Guez de Balzac. L'histoire littéraire semble donc être le domaine de prédilection de Drouhet qui en assimile non seulement les méthodes, mais aussi un certain éthos. Selon Munteanu, « on le sent tourmenté par une véritable soif de précision, par un terrible besoin de netteté, de rigueur, de certitude en un mot, qui, chez ce pur historien des lettres, peu enclin aux jeux, même féconds, de la philosophie, tiennent lieu et place de toute autre forme d'inquiétude » (Munteanu 1940 : 16). Cette attitude par rapport à la connaissance s'ajoute aux approches qu'il met en œuvre dans ses recherches et invitent à une 
comparaison entre lui et son précurseur dans le poste de professeur de littérature française à l'Université de Bucarest, Pompiliu Eliade.

Comme lui, mais dix ans auparavant, Eliade avait défendu ses thèses à l'Université de Paris. Mais là où Drouhet fait un travail de recherche très minutieux et fondé sur la critique des sources, Eliade avait fait plutôt œuvre d'historien « romantique », dans son ouvrage intitulé De l'influence française sur l'esprit public en Roumanie (Eliade 1898). Inspiré par les grandes constructions d'un Hyppolite Taine et d'un Ferdinand Brunetière, Eliade arrivait à Paris lorsque leurs approches étaient contestées par les nouvelles générations d'historiens et s'attirait, lors de la soutenance, les critiques de Charles Seignobos, auteur d'une Introduction aux études historiques (Langlois \& Seignobos 1898) prise comme modèle pour les études littéraires par Gustave Lanson. Dix ans plus tard, à l'époque où Drouhet soutient sa thèse, la méthode de Seignobos et Lanson s'était imposée, atteignant le statut de "science normale », et les deux auteurs étaient devenus deux piliers de la « République des universitaires » (Charle 1994). Dans ce contexte, pour le jeune chercheur inscrit en doctorat à la Sorbonne, ce que Pierre Bourdieu appelle «l'espace des possibles » (voir Bourdieu 1984) s'était considérablement rétréci. Le fait qu'il était étranger, ce que Gustave Lanson mentionne pas moins de trois fois dans son compte-rendu, contribuait à réduire sa marge de manœuvre. Mais, pour Drouhet, ce choix a aussi une signification qui relève de l'espace roumain de la discipline. C'était une manière de se démarquer de son précurseur Pompiliu Eliade, une différence explicitée très nettement dans l'article écrit en hommage à la mort de celui-ci, en 1914. Agissant entre la Roumanie et la France, en d'autres mots entre une culture périphérique et une culture majeure, les premières recherches de Drouhet adhèrent à des principes qui favorisent la modernisation des études littéraires.

Les sujets de la « seconde étape » se rattachent à une autre approche qui, en reprenant une des méthodes de l'histoire littéraire (la critique des sources), était en cours de définition dans le champ académique français de l'époque : la littérature comparée. Depuis la fin du XIX ${ }^{\mathrm{e}}$ siècle, avec Joseph Texte et la création de la première chaire à Lyon en 1897, la littérature comparée s'était développée comme un cadre qui permettait de traiter les relations entre littératures nationales, d'abord entre les grandes littératures européennes et ensuite, progressivement, entre les grandes et les petites littératures (Van Tieghem 1931). Cette approche permet ainsi à Drouhet d'étudier les rapports entre la littérature française et la littérature roumaine, par le biais des études d'influences. Son autre grand ouvrage (Drouhet 1924), qu'il commence en 1911 et qu'il publie finalement en 1924, porte sur l'écrivain roumain qui a eu les plus fortes relations avec la France au XIX ${ }^{\mathrm{e}}$ siècle, le poète Vasile Alecsandri (1821-1890), et sur les influences qu'il a pu subir de la part des romantiques français (Victor Hugo, Lamartine, etc.). En suivant une ligne ouverte par Pompiliu Eliade, avec son intérêt pour les influences 
françaises dans l'espace roumain, Drouhet se démarque toutefois de celui-ci, en accordant au poète Alecsandri le droit à l'originalité par rapport à ses maîtres : " L'inspiration religieuse, philosophique et sociale de son illustre initiateur en poésie [Lamartine] ne trouvera pas d'écho dans son âme. Alecsandri ne suivit pas non plus Victor Hugo, jusqu'au terme de son évolution littéraire et n'imita pas tous les aspects de son génie » (voir Drouhet 1922 : 487).

Identifier les possibles sources de ces poèmes est donc pour Drouhet une étape essentielle dans sa démarche d'historien littéraire. Toutefois si, à l'époque de ses études parisiennes, il souhaite se légitimer par des publications dans la Revue d'histoire littéraire de la France, après la Grande Guerre c'est la nouvelle Revue de littérature comparée, fondée en 1921, qui attire son attention. Cette fois, il sera présent grâce à son disciple, Basil Munteanu, qui y publiera trois recensions de ses recherches sur les sources françaises des écrivains roumains Alecsandri, Gr. Alexandrescu et Konaki. Le souci de garder les relations avec la communauté des chercheurs français se voit également dans les lettres qu'il envoie à Munteanu, à Paris, pour lui demander les nouvelles publications et pour lui faciliter les contacts avec les professeurs de la Sorbonne et du Collège de France.

Ses études sur Alecsandri ont ensuite été critiquées, comme tout ce qui a été produit sous l'influence du comparatisme français de l'entre-deux-guerres. Cette démarche serait utile pour évoquer plutôt l'atmosphère littéraire d'une époque, les thèmes et motifs présents dans l'air du temps, et elle ne peut pas prouver « scientifiquement», empiriquement, l'influence qu'on entend démontrer. Ce genre de reproches, faits dans les années 1970, se situe toutefois sur une position normative, qui ne tient pas compte des critères de validité de l'époque de Drouhet. En effet, dans sa démarche, il ne fait que suivre les suggestions qui avaient été développées, entre autres, par Fernand Baldensperger dans son Goethe en France (1904), et qui seront par la suite exploitées dans les numéros de la Revue de littérature comparée. Vu que la littérature d'un pays était considérée comme une sous-espèce de la littérature universelle, la tâche du comparatiste était de restituer les fertilisations croisées et les mutations que ces rencontres avaient produites. Par ses études sur Alecsandri et les romantiques français, Drouhet s'inscrivait ainsi dans un réseau de recherche transnational qui partageait les mêmes critères de validité et les mêmes objectifs.

\section{Quelle littérature française pour l'université roumaine?}

L'activité de professeur à l'Université de Bucarest commence pour Charles Drouhet en 1915, après la mort de son prédécesseur, Pompiliu Eliade. Titulaire, depuis son doctorat, de la chaire de Langue et littérature françaises à l'Université 
de Jassy, Drouhet profite de la vacance du poste dans l'université de la capitale et s'inscrit ainsi dans un mouvement plus général des professeurs de province pour lesquels un tel transfert était autant une promotion scientifique, que son doctorat à Paris justifiait, qu'une promotion sociale. Il y restera jusqu'à sa mort, en 1940.

Prendre pour objet d'étude son enseignement signifie ainsi s'intéresser à « la littérature française » en tant que discours qu'il construit et transmet à ses étudiants (pour une histoire du cours magistral, voir Bruter 2008). Mais, pour restituer le sens de cette construction, plusieurs questions s'imposent. Tout d'abord, quels sont les éléments qui composent ce discours, les écrivains et les œuvres dignes d'être présentés aux étudiants ? En d'autres mots, il s'agit de voir « la littérature française » de Drouhet comme le résultat d'une sélection à ambition canonisante et pour laquelle il faut retrouver non seulement la tension entre les présents et les absents, mais aussi les critères qui justifient la sélection et les appréciations que le professeur porte sur ces écrivains. En même temps, la sélection et les appréciations font partie de formes discursives plus générales, qu'il est important de mettre au jour, parce qu'elles orientent le sens donné aux discussions des cas individuels.

À regarder la matière des cours qu'il a donnés entre 1915 et 1940 et qui ont été publiés annuellement à l'intention des étudiants, la « littérature française » selon Drouhet se compose de mouvements littéraires successifs, qui ne s'identifient pas aux "siècles littéraires », tels qu'ils avaient été définis par les historiens de la littérature du XIX ${ }^{e}$ siècle. Dans l'introduction du dernier cours (Drouhet 1940), il donne comme critère de division les « événements littéraires », la publication de certains ouvrages, chefs-d'œuvre de la littérature, qui ont déterminé un changement dans le style ou dans les préoccupations des écrivains. Ces points d'inflexion ont fourni à Drouhet la matière de ses enseignements : chaque année académique est consacrée à la présentation d'un " mouvement littéraire », que ce soit le romantisme, le préromantisme ou le réalisme, ou d'un genre représentatif (le théâtre du XVII ${ }^{\mathrm{e}}$ siècle, le roman réaliste). Ainsi, le cours magistral construit une histoire de la littérature française, qui commence habituellement par la Renaissance et qui finit par le réalisme, tout cela répété approximativement trois fois pendant plus d'une vingtaine d'années. Par exemple, le mouvement littéraire à l'époque du réalisme constitue le sujet du cours de 1921-1922, ensuite du cours de 1930-1931 et finalement du cours de 1938-1939.

Chaque cours est composé habituellement d'une vingtaine de leçons ; il commence par une introduction sur l'époque et les courants d'idées dominants (éventuellement sur les contextes sociaux particuliers, comme les salons au XVIII ${ }^{\mathrm{e}}$ siècle) et continue ensuite par une série de discussions des écrivains représentatifs du mouvement littéraire concerné et de leurs œuvres. Bien que l'auteur évoque un nombre assez important d'écrivains en connexion avec un certain mouvement, les cours semblent néanmoins être construits autour des grands écrivains d'une 
époque : ainsi, le cours sur la littérature des philosophes (la première partie du XVIII ${ }^{\mathrm{e}}$ siècle) donné en 1925-1926 comporte trois leçons sur Montesquieu et deux leçons sur Voltaire; le cours sur le réalisme de 1938-1939 comporte six leçons sur Flaubert (Drouhet 1938). Parfois, au critère d'organisation de la matière en fonction des écrivains s'ajoute celui des genres littéraires, plusieurs leçons portant par exemple sur le roman dans la première partie du XVIII ${ }^{\mathrm{e}}$ siècle.

Cette histoire de la littérature française par cours successifs est faite dans le cadre de pensée habituel (depuis le XIX ${ }^{\mathrm{e}}$ siècle) de la littérature vue comme expression du génie du peuple, les œuvres des grands écrivains étant considérées comme les manifestations les plus parfaites de l'esprit de la nation et de la race, dont l'influence sur le monde contemporain est encore visible. C'est ainsi que, par exemple, les idées des philosophes du XVIII ${ }^{\mathrm{e}}$ siècle, qui ont donné naissance à la Révolution française, vivent encore dans les esprits et, selon Drouhet, il n'est pas un hasard si le président américain Wilson, celui qui a apporté la paix mondiale, avait été imprégné des idées de Montesquieu. Mais cette histoire de la littérature se construit aussi en fonction de certains jugements de valeur et Drouhet n'hésite pas à reconnaître qu'il présente l'époque surtout dans ses « traits positifs », sans s'attarder sur les mauvais poèmes d'un André Chénier, sur l'érudition prolixe d'un Pierre Bayle, sur les récits ennuyeux de Marivaux ou sur les fautes de La Rochefoucauld.

La formation du professeur fait que son enseignement en Roumanie soit comparable à l'enseignement qu'on donnait à la même époque en France. Il n'hésite cependant pas à faire des références fréquentes aux écrivains roumains qui ont été influencés ou qui ont traduit les écrivains français dont il est question. De cette manière, il essaie de situer la littérature française par rapport à l'horizon culturel de l'élève roumain qui, tout en étudiant de manière approfondie la littérature française (voir ci-dessous), n'était pas dans la même position que l'élève français.

Chaque année, le cours principal était accompagné d'un « cours de commentaires », dans lequel Drouhet se consacrait à la présentation détaillée d'un seul écrivain. Ainsi, Montaigne, Boileau, Voltaire, La Fontaine, Rabelais, Villon ont été analysés en profondeur par la méthode de l'explication de texte que l'auteur avait apprise auprès de Gustave Lanson (voir Lanson 1925) et qu'il s'employait à introduire en Roumanie. Ce type de lecture "rapprochée », dont le but était d'éclairer les significations du texte à tous les niveaux, devenait avec Drouhet une compétence obligatoire pour l'étudiant qui cherchait une formation dans la littérature française et dans la philologie moderne. ${ }^{3}$

\footnotetext{
${ }^{2}$ Pour l'étudiant qui souhaitait obtenir la licence en philologie moderne à la Faculté de Philosophie et Lettres de l'Université de Bucarest, le règlement de 1924 exigeait sur les trois années d'étude au moins six examens passés, au choix, dans les disciplines suivantes : Philologie romane et langue roumaine, Histoire de la littérature roumaine, Littérature roumaine et esthétique littéraire, Langue
} 


\section{Former le futur citoyen roumain par la littérature française?}

Un dépouillement des catalogues des bibliothèques roumaines, à la recherche des écrits de Charles Drouhet, offre parmi les résultats, à l'exception des ouvrages de critique et d'histoire littéraire évoqués ci-dessus, un grand nombre de manuels de français réalisés par le professeur et ses collaborateurs durant les années 1920-1930 et réédités à plusieurs reprises, même pendant les années 1940, après la mort de l'auteur. Le catalogue de la Bibliothèque de l'Académie roumaine de Bucarest enregistre 87 manuels écrits par Drouhet (y compris les rééditions) et seulement 3 manuels écrits par Nicolae Șerban, l'autre professeur universitaire de français du "Vieux Royaume », à l'Université de Jassy. Cela montre à quel point son influence a été grande parmi les professeurs du secondaire (ses anciens étudiants), qui utilisaient ses ouvrages dans la formation des élèves des lycées. Pour découvrir les enjeux de la littérature française dans l'enseignement secondaire roumain de l'époque, ces manuels représentent un corpus incontournable, d'autant plus qu'en 1936-37, l'auteur en a donné une édition complète (des ouvrages pour toutes les classes de l'enseignement secondaire, de la première à la huitième). Mais, pour comprendre ces enjeux, il faudrait resituer les manuels dans le contexte de leur production, parce qu'il s'agit d'ouvrages qui répondent à des textes officiels, les programmes pour l'enseignement secondaire. Cette partie se propose ainsi de discuter le programme de français dans son évolution historique jusqu'aux années 1930, ensuite le rôle de Charles Drouhet dans l'élaboration des textes officiels de l'époque et enfin les manuels qu'il élabore en réponse à ces programmes. Derrière cette répartition on pourra retrouver, nous l'espérons, la constitution d'un discours sur les objectifs de l'enseignement de la littérature française pour la formation du jeune élève de la Grande Roumanie.

Publiés dans le Moniteur officiel, les programmes représentent les normes d'application de la législation de l'enseignement secondaire et détaillent, pour chaque discipline, les objectifs, les méthodes et les contenus spécifiques de chaque classe (pour une réflexion sur les programmes d'enseignement, voir Demonque 1994). En ce qui concerne les objectifs, une évolution à deux niveaux est visible : d'une part, si dans la seconde moitié du XIX ${ }^{\mathrm{e}}$ siècle (les premiers textes officiels datent de 1864), les programmes n'avaient pas de fins explicites, à partir de 1899 (avec les réformes de Spiru Haret) on constate une réflexion de plus en plus éla-

et littérature françaises, allemandes, italiennes, latines, Philologie comparée des langues classiques, Philologie slave, Dialectologie et folklore, Histoire des Roumains, Histoire universelle, Histoire des arts, Pédagogie. Les autres parcours de licence possibles étaient : Philosophie, Philologie classique, Histoire et géographie. En 1926-1927, la Faculté comptait 4725 étudiants inscrits, dont 2 141 dans la section Philologie moderne (toutes années confondues). 416 étudiants (toutes sections confondues) ont obtenu leur licence en 1926-1927 (Popescu-Spineni 1928). 
borée sur le rôle de la discipline ; d'autre part, cette réflexion transforme l'enseignement du français d'une activité dont le but est la connaissance de la langue en une activité qui vise non seulement la conversation et la capacité d'écrire et de lire, mais aussi la compréhension des beautés esthétiques des œuvres littéraires.

Pour ce qui est des « contenus », les textes indiquent d'habitude des noms d'auteurs et parfois des titres d'ouvrages qui doivent constituer l'objet des leçons. Si le programme de 1864 évoque un nombre restreint d'auteurs qu'on pourrait considérer comme « classiques » (Racine, Boileau, Molière, Rousseau, Corneille, Voltaire), les textes successifs voient un élargissement considérable, tant du point de vue du nombre, que de celui des époques littéraires étudiées. La tendance qu'on peut observer est l'apparition de l'idée d'histoire de la littérature, avec une répartition des écrivains pour chaque classe du lycée. À titre d'exemple, le programme de 1934 (voir Monitorul oficial 1934) consacre la vle classe au classicisme (de Corneille à Voltaire), la VII elasse au préromantisme et au romantisme (de Rousseau à Vigny) et la dernière classe de lycée au réalisme, au naturalisme et à la réaction contre ceux-ci (de Balzac à Taine). Toute cette succession était précédée par un panorama général sur le développement de la littérature française, en $v^{\mathrm{e}}$.

Les manuels de Drouhet sont élaborés bien sûr en concordance avec les documents du ministère, mais les choix de la liste officielle des auteurs et des œuvres lui appartiennent, tout comme les " sujets de conversation », qui permettent de voir comment un extrait était censé être lu par l'élève. En analysant cette sélection, ainsi que le paratexte pédagogique (explications, sujets de conversation et de devoirs), on peut découvrir une pensée plus nuancée de la littérature française, qui est celle de l'auteur, et non pas du programme officiel. Le canon scolaire se forme ainsi à l'intersection des règles institutionnelles et des décisions individuelles de l'auteur.

Dans le manuel de $v^{e}$ (Drouhet \& Ghiacioiu 1937), on peut discerner une tendance à développer le sens pratique de l'élève. Plusieurs extraits sont accompagnés des questions comme « Est-ce que ce fragment a une signification morale ? Sinon, quel est son intérêt ? ». La sélection semble être faite de manière à transmettre l'idée que l'élève doit s'adapter aux circonstances et non pas suivre des idéaux utopiques. Les fables de La Fontaine constituent une partie importante du manuel et elles sont accompagnées des suggestions sur l'écart entre la réalité et le rêve (La laitière et le pot au lait) ou sur l'esprit pratique nécessaire dans la vie (Le Héron). Les autres textes sont choisis selon le même critère : avec Montesquieu (le fragment « Comment peut-on être Persan ? »), l'élève peut se moquer de la curiosité extravagante des Parisiens, avec Rabelais (« les moutons de Panurge ") l'élève doit se garder de "l'imitation irréfléchie (la routine) 》. Cette présentation générale de la littérature française semble ainsi être faite en accord avec l'idée utilitariste sur l'éducation secondaire, par laquelle l'élève doit acquérir une perspective appliquée sur la vie. 
Les autres manuels, consacrés aux périodes de la littérature française, ajoutent des remarques littéraires sur les idées, le style et la composition des extraits, mais l'auteur n'abandonne pas le souci pratique. Ainsi, avec Rousseau (Émile), on célèbre le travail manuel, et avec Flaubert on critique Madame Bovary parce qu'elle ne rêve pas du Paris des travailleurs ou des étudiants (« Les seules classes qui l'intéressent sont celles de désœuvrés et des fêtards », Drouhet 1937). Pour ce qui est du canon, son histoire de la littérature va jusqu'aux poètes symbolistes, mais sans mentionner Baudelaire, peu conforme à l'éducation des élèves de cet âge. Les ouvrages de Drouhet réussissent à compléter l'intérêt pour l'éducation (les valeurs que l'élève était censé acquérir) avec celui pour l'instruction (le savoir sur le style des différents écrivains). Il se situe ainsi sur une position modéré dans le débat sur le rôle de l'éducation secondaire qui caractérise le discours pédagogique de l'époque, en France comme en Roumanie (Jey 1998).

La modération de Drouhet est visible aussi dans un autre débat qui traverse toute l'éducation de la Roumanie de l'entre-deux-guerres, sa mission nationale. Selon Irina Livezeanu, pendant les années 1920-1930, l'éducation roumaine avait pour fin, à tous les niveaux, la consolidation de la nation (Livezeanu 1995). Suite au Traité de Versailles, qui mettait fin à la Grande Guerre, des territoires importants avec une population roumaine ont été intégrés dans le Vieux Royaume et ce peuple devait être éduqué dans l'esprit national roumain. La littérature roumaine, l'histoire et la géographie étaient bien sûr les disciplines les plus importantes, mais aucune autre n'était exclue et la littérature française devient elle aussi, de manière assez paradoxale, un instrument dans le processus de consolidation de l'identité nationale.

$\mathrm{Si}$ on analyse les programmes officiels de la littérature française, on peut observer comment cette dimension, pratiquement absente au XIX $x^{\mathrm{e}}$ siècle, apparaît de manière de plus en plus évidente. Le texte de 1934 stipule que les manuels de $I V^{\mathrm{e}}$ et $\mathrm{V}^{\mathrm{e}}$ devront contenir « des morceaux des voyageurs et écrivains français concernant la Roumanie (par exemple : Michelet : Roumanie, Nation sacrifiée, Ed. Quinet : L'idée d'une grande nation » [voir Monitorul oficial 1934]). Des activités supplémentaires peuvent être consacrées à des morceaux écrits par des écrivains français concernant le peuple roumain : "Ces écrits ouvriront de nouveaux horizons aux élèves, leur présentant le propre pays par un regard de l'Ouest, un regard qui a des orientations et des intérêts différents. À cette occasion, on discutera la situation de notre pays en rapport étroit avec l'époque culturelle concernée » (voir Monitorul oficial 1934).

Cette contextualisation nationale n'est en fait que la dernière étape d'une controverse plus longue sur l'enseignement du français, qui occupe le début des années 1930. Dans la Revue générale de l'enseignement, Charles Drouhet prend position en 1932 par rapport aux projets de programmes qui venaient d'être rendus publics par le Ministère (Drouhet 1932). Son texte, assez exceptionnel si on 
le compare avec d'autres articles que la revue publiait à l'époque, est une critique des présupposés nationaux qui formaient la base de l'enseignement du français dans la vision des nouveaux textes officiels. Suivant l'idée que, pour apprendre le français, on devait partir des réalités du pays et de la civilisation roumains, le projet de programme souhaitait que les élèves étudient les textes des auteurs roumains du XIX ${ }^{\mathrm{e}}$ siècle ayant écrit en français. L'erreur, selon Drouhet, était de ne pas se rendre compte que la langue était l'expression d'une civilisation et que ce qui était propre à Bucarest ou aux provinces roumaines n'avait pas de correspondant à Paris ou dans les provinces françaises. Apprendre le français des écrivains roumains était comme apprendre le latin des auteurs de la Renaissance, au lieu de lire les écrits d'Ovide, Virgile ou Horace. Ce programme partait d'une erreur de principe et cela, selon Drouhet, parce qu'il avait été conçu dans les bureaux du ministère, sans demander l'avis des enseignants. Son article critique semble avoir eu un effet, parce que le programme de 1934, comme on vient de le voir, est plus modéré dans son projet national. La position de Drouhet peut ainsi être vue comme une contribution à l'autonomie de la discipline, dans un contexte d'interférences politiques.

Ses manuels suivent les recommandations, en utilisant par exemple, pour la classe de $\mathrm{v}^{\mathrm{e}}$, trois extraits de Michelet, historien français qui a admiré la lutte des roumains pour l'indépendence (Drouhet \& Ghiacioiu 1937 : 173-181). Les sujets de conversation ( $Q$ Quels sentiments l'auteur éprouve-t-il pour la Roumanie ? Quels sentiments éprouvons-nous à notre tour pour cet étranger qui parle avec tant de sympathie de notre pays ? ») ont pour but de situer la culture nationale dans une relation d'empathie avec la culture française et l'élève apprend ainsi que la littérature française n'est pas n'importe quelle littérature, mais celle d'une nation amie de la Roumanie. Toutefois, ces suggestions ne sont pas très fréquentes, surtout si on compare ce manuel à celui d'un autre auteur, Nicolae Şerban, professeur de l'Université de Jassy. Les manuels de Drouhet sont ainsi le résultat d'une conception modérée de l'enseignement de la littérature française au lycée, dans un contexte où l'éducation à tous les niveaux dans la Grande Roumanie avait pour but la consolidation de la nation.

\section{Conclusions}

L'activité de Drouhet a laissé des traces importantes. Son intérêt pour les relations entre la littérature française et la littérature roumaine a été poursuivi par ses successeurs. Dans les années 1970, des thèses importantes faites par des enseignants de l'Université de Bucarest ont été consacrés aux « fortunes » de Rousseau et de Balzac en Roumanie. Dans les vingt dernières années, des écrivains d'origine roumaine ayant écrit en français, comme Panait Istrati ou Emil 
Cioran, constituent des sujets de recherche légitimes, bien que situés dans une perspective d'anthropologie culturelle. Même si la vague structuraliste venue de France a beaucoup contribué à la modernisation des recherches sur la littérature française en Roumanie, l'histoire littéraire reste encore en arrière-plan comme un horizon méthodologique pertinent pour les études littéraires. Du point de vue de l'activité universitaire, Drouhet se situe à l'origine de deux pratiques encore très répandues, l'enseignement de la littérature française par époques, et l'explication de texte comme exercice pour les étudiants. En revanche, ce qui s'est un peu perdu, c'est la dimension nationale de l'enseignement de la littérature étrangère. Si cette dimension était encore très présente à l'époque du national-communisme, les dernières décennies ont vu l'introduction du paradigme communicationnel dans l'enseignement des langues étrangères. De plus, le français n'est plus la première langue étrangère étudiée en Roumanie et la littérature française n'est presque plus étudiée au lycée. On peut dire ainsi que la littérature française a perdu, dans l'enseignement secondaire, le statut patrimonial traditionnel et les enseignants d'aujourd'hui doivent s'adapter à de nouvelles logiques de fonctionnement de la discipline. Revisiter son histoire permet ainsi de comprendre les effets de continuité sur la longue durée, ainsi que les transformations provoquées par les facteurs internes et externes.

\section{Références bibliographiques}

Bourdieu 1984 : P. Bourdieu, Homo academicus, Paris : Minuit.

Bruter 2008 : A. Bruter, « Le cours magistral comme objet d'histoire » in Histoire de l'éducation, $\mathrm{n}^{\circ}$ 120, 5-32.

Charle 1994 : Ch. Charle, La République des universitaires, Paris : Seuil.

Compagnon 1983 : A. Compagnon, La Troisième République des Lettres, de Flaubert à Proust, Paris : Seuil.

Demonque 1994 : Ch. Demonque (dir.), Qu-est'ce qu'un programme d'enseignement?, Paris : Hachette éducation.

Drouhet 1909 : Ch. Drouhet, Le poète François Mainard (1583 ?-1646). Etude critique d'histoire littéraire, Paris : Honoré Champion.

Drouhet 1922 : Ch. Drouhet, « De l'influence française dans la poésie de Basile Alecsandri », in Mélanges offerts par ses amis et élèves à M. Gustave Lanson, Paris: Hachette, 479-487.

Drouhet, 1924 : Ch. Drouhet, Vasile Alecsandri și scriitorii francezi, București : Cultura națională.

Drouhet 1932 : Ch. Drouhet, « Programele analitice de limba franceză din 1932 » in Revista generală a învățământului, no 9-10, 61-71.

Drouhet \& Ghiacioiu 1937 : Ch. Drouhet \& V. Ghiacioiu, Extraits des auteurs français depuis les origines de la littérature française jusqu'à nos jours, à l'usage des élèves de la $V^{o}$ classe des lycées roumains, Bucarest : Naționala-Ciornei. 
Drouhet 1938 : Ch. Drouhet, Literatura franceză. Realismul, București : Facultatea de filosofie și litere.

Drouhet 1940 : Ch. Drouhet, Literatura franceză. Preclasicismul francez, București : Facultatea de filosofie și litere.

Fabiani 2006 : J.-L. Fabiani, « À quoi sert la notion de discipline ? » in J. Boutier, J.-Cl. Passeron, J. Revel, Qu'est-ce qu'une discipline ?, Paris : Ed. de l'EHESS, 11-34.

Eliade 1898 : P. Eliade, De l'influence française sur l'esprit public en Roumanie, Paris : Ernest Leroux.

Jey 1998 : M. Jey, La littérature au lycée : invention d'une discipline (1880-1925), Metz : Université de Metz.

Jipa 2018 : D. Jipa, « Faire l'histoire de la "littérature française” comme discipline académique dans la Roumanie moderne (1864-1948). Considérations théoriques et méthodologiques » in New Europe College Yearbook 2017-2018, Bucarest, 151-174.

Langlois \& Seignobos 1898 : Ch.-V. Langlois, Ch. Seignobos, Introduction aux études historiques, Paris : Hachette.

Lanson 1910 : G. Lanson, « Compte-rendu », Revue d'histoire littéraire de la France, $\mathrm{n}^{\circ}$ 2, Paris : PUF, 403-405.

Lanson 1925 : G. Lanson, Méthodes de l'histoire littéraire, Paris : Les Belles Lettres.

Livezeanu 1995 : I. Livezeanu, Cultural politics in Greater Romania: regionalism, nation building and ethnic struggle, 1918-1930, Ithaca: Cornell University Press.

Monitorul oficial 1934 : Monitorul oficial, no 191.

Munteanu 1940 : B. Munteanu, «Ch. Drouhet. Le Savant - Le Professeur » in Mélanges d'histoire littéraire et de littérature comparée offerts à Charles Drouhet par ses amis, ses collègues et ses anciens élèves, Bucarest.

Popescu-Spineni 1927 : M. Popescu-Spineni, Contribuțiuni la istoria învățământului superior. Facultatea de filosofie și litere din București, București : Cultura națională.

Schaeffer 2011 : J.-M. Schaeffer, Petite écologie des études littéraires, Paris : Thierry Marchaisse.

Țurcanu 2006 : F. Țurcanu (dir.), Modèles français et expériences de la modernisation, Bucarest : ICR.

Van Tieghem 1931 : P. Van Tieghem, La Littérature comparée, Paris : Armand Colin. 


\section{Dragoš Žipa}

\section{Francuska književnost kao univerzitetska disciplina u Rumuniji između dva rata: slučaj Šarla Druea}

U radu analiziramo proučavanje i podučavanje francuske književnosti u Rumuniji u međuratnom periodu, tumačeći s jedne strane osnovne koncepcije ustanovljene discipline i sagledavajući je, s druge strane, u institucionalnim i (među)nacionalnim okvirima epohe. Polazeći od primera Šarla Druea, profesora na Univerzitetu u Bukureštu, nastojaćemo da pokažemo kako su discipline istorije književnosti i komparativne književnosti, „uvezene“ u Rumuniju iz Francuske, postavile metodološke temelje i kriterijume naučnog istraživanja i predavanja francuske književnosti na univerzitetu. Pored toga, uočljivo je da pred namerom učvršćenja nacionalnog jedinstva putem predavanja francuske književnosti u srednjoj školi Šarl Drue zastupa nezavisnost znanja od upliva političkih faktora. Posmatran iz današnje perspektive, ovaj istorijat discipline francuske književnosti u Rumuniji omogućuje bolje razumevanje etapa razvoja ove oblasti do kraja XX veka.

Ključne reči: francuska književnost, podučavanje, naučno istraživanje, institucionalizacija, književna istorija, komparativna književnost, nacija. 